\title{
Multicriteria Ranking Using Weights Which Minimize the Score Range
}

\section{CHRIS TOFALLIS}

\author{
University of Hertfordshire Business School Working Paper (2010) \\ University of Hertfordshire Business School Working Papers are available for download \\ from tinyurl.com/workingpapers \\ and also from the British Library: www.mbsportal.bl.uk
}

The Working Paper Series is intended for rapid dissemination of research results, work-in-progress, and innovative teaching methods, at the pre-publication stage. Comments are welcomed and should be addressed to the individual author(s). It should be noted that papers in this series are often provisional and comments and/or citations should take account of this.

Copyright and all rights therein are retained by the authors. All persons copying this information are expected to adhere to the terms and conditions invoked by each author's copyright. These works may not be re-posted without the explicit permission of the copyright holders.

The Business School at the University of Hertfordshire (UH) employs approximately 150 academic staff in a state-of-the-art environment located in Hatfield Business Park. It offers 17 undergraduate degree programmes and 21 postgraduate programmes; there are about 80 research students, mostly working at doctoral level.

Business School staff are active in research in numerous areas, including complexity theory, institutional economics, economic modelling, efficiency measurement the creative industries, employment studies, finance, accounting, statistical methods and management science.

The University of Hertfordshire has been recognised as the exemplar of a business-facing university. It is one of the region's largest employers with over 2,700 staff and a turnover of $£ 235$ $\mathrm{m}$.

In the 2008 UK Research Assessment Exercise it was given the highest rank for research quality among the post-1992 universities. 


\title{
Multicriteria Ranking Using Weights \\ Which Minimize the Score Range
}

\author{
Dr. Chris Tofallis \\ The Business School \\ University of Hertfordshire \\ College Lane \\ Hatfield \\ Hertfordshire \\ AL10 9AB
}

\section{email: c.tofallis@herts.ac.uk}

A version of this paper appears in

New Developments in Multiple Objective and Goal Programming,

D. Jones et al. (eds.), 2010

Lecture Notes in Economics and Mathematical Systems 638,

DOI 10.1007/978-3-642-10354-4 9,

Springer-Verlag, Berlin, Heidelberg.

Note: An early version of part of this paper was presented at the EWEPA conference in Lille. It was awarded a prize by ballot of the participants.

\begin{abstract}
Various schemes have been proposed for generating a set of non-subjective weights when aggregating multiple criteria for the purposes of ranking or selecting alternatives. The maximin approach chooses the weights which maximise the lowest score (assuming there is an upper bound to scores). This is equivalent to finding the weights which minimize the maximum deviation, or range, between the worst and best scores (minimax). At first glance this seems to be an equitable way of apportioning weight, and the Rawlsian theory of justice has been cited in its support.

We draw a distinction between using the maximin rule for the purpose of assessing performance, and using it for allocating resources amongst the alternatives. We demonstrate that it has a number of drawbacks which make it inappropriate for the assessment of performance. Specifically, it is tantamount to allowing the worst performers to decide the worth of the criteria so as to maximise their overall score. Furthermore, when making a selection from a list of alternatives, the final choice is highly sensitive to the removal or inclusion of alternatives whose performance is so poor that they are clearly irrelevant to the choice at hand.
\end{abstract}




\section{Introduction}

One of the most influential works in the area of moral and political philosophy in the last 50 years has been John Rawls's A Theory of Justice (1971). Rawls rejects the utilitarian idea of 'the greatest good for the greatest number'. This is a concept which the decision science community would recognize as being fraught with difficulties. These include the fact that 'the good' is likely to be a multi-factor concept, and that we are also dealing with multiple stakeholders holding different views. It is important to note that even if there were agreement on how to measure and then aggregate the overall good of the population, it does not follow that maximizing it would provide any form of social justice unless of course such justice was built into the definition of 'the good'. Rawls viewed "justice as fairness" and felt that the worst off should not be made even worse. In particular, if public resources are to be distributed unequally, then the worst off should benefit the most. Rawls referred to this as the 'difference principle'.

Rawls has been cited in support of using the maximin rule for weighting criteria by Pettypool and Karathanos (2004). They proposed the rule for the purpose of appraising the work of employees under a number of criteria. Butler and Williams (2002) use the maximin rule in sharing out the fixed costs associated with shared facilities. In support of it they cite work based on experiment and survey:

'A variety of fairness criteria are discussed in the seminal paper of Yaari and Bar-Hillel (1984). They conducted a series of experiments to see which of nine possible criteria were considered most fair by a sample of people questioned. In relation to needs, an allocation based on minimizing the maximum inequality was overwhelmingly considered the most fair.'

The allocation of resources is another area where this rule has been proposed. Ogryczak and Sliwinski (2002) assert that the maximin rule is consistent with Rawlsian theory of justice but point out that "allocating the resources to optimize the worst performances may cause a large worsening of the overall (mean) performances". One example of a practical application of the maximin objective was to allocate highway patrol officers to districts so as to ensure that all districts experience a reduction in speeding; the aim was to maximize the minimum reduction in the number of speeding offences (Rardin, 1998, p.158). In the field of job scheduling numerous objectives are employed, one of these is to minimize the maximum lateness (Rardin, 1998, p.605). It is also used to minimize maximum congestion or bottlenecks. Du (1996) surveys the field of minimax applications.

One field where the minimax concept is widely used is in location problems (Farahani, 2010). When choosing locations for emergency facilities (police, ambulance, fire- 
fighting) or other public offices or services, this method selects locations so as to minimize the maximum travel time or distance to any person who is being served. The method has been criticized (e.g. Ogryczak, 1997) because if there is a single recipient (or a small cluster) that is located far from the vast majority, then a location may be selected which is far from all recipients. There is thus seen to be a disproportionate effect on the decision by a tiny minority of the recipients. We shall see that a similar difficulty arises when applying the minimax concept to multicriteria weighting.

\section{Applications of the maximin principle for weight estimation}

In multi-criteria analysis the objects being compared are referred to as 'alternatives', whilst in the field of efficiency analysis they tend to be called DMUs (decision making units). The maximin concept has been used in the assessment of performance by a number of authors. For example, Karsak and Ahiska (2005) and Karsak (2004) consider the problem of attaching a common set of weights to the various outputs (i.e. criteria of the type 'more is better') when there is a single input. To create an efficiency score each output is weighted and then divided by the input. In DEA (data envelopment analysis) each alternative (or Decision Making Unit) being compared has its own weights. These are chosen so as to optimize the score for that alternative. Because this method attaches different weights for each alternative, this leads to the generation of an efficient frontier which is made up of piecewise linear segments. In DEA all the alternatives on the frontier are given the same score of $100 \%$. When there are many dimensions the frontier will have many such observations and it is sometimes felt that there is a lack of discrimination. In an effort to increase the discrimination between such units and identify a preferred alternative, the above authors sought a way of generating a common set of weights to be used across all alternatives. These non-negative weights are chosen so as to maximize the minimum score (maximin), subject to the condition that all scores do not exceed $100 \%$. In criterion space a set of common weights corresponds to a line or plane.

Figure 1 shows an example involving two criteria. According to DEA, points A, B and C are ranked first with the maximum score, and $\mathrm{ABC}$ delineates the DEA frontier. In DEA alternative $\mathrm{P}$ has a score given by the ratio $\mathrm{OP} / \mathrm{OP}^{\prime}$, where $\mathrm{P}^{\prime}$ is the point where the ray $\mathrm{OP}$ intersects the frontier. Because $\mathrm{P}^{\prime}$ lies between $\mathrm{A}$ and $\mathrm{B}$, the corresponding weights are determined by the slope of the line AB. Point $\mathrm{T}$ however would be assessed relative to the line segment $\mathrm{BC}$, which corresponds to a different set of criteria weights. Of the points shown in Figure 1, P would have the lowest score. If we now depart from the piecewise frontier in favour of a single set of common weights based on the maximin rule, we shall have a single extended line frontier. We shall have to choose weights which maximize P's score, and so the frontier will be AB (extended). Notice that the particular line segment and hence weights, are chosen by reference to the worst performing alternative. This in itself is strange because the frontier is supposed to represent best practice, and yet its location is crucially influenced by an alternative displaying worst practice. 


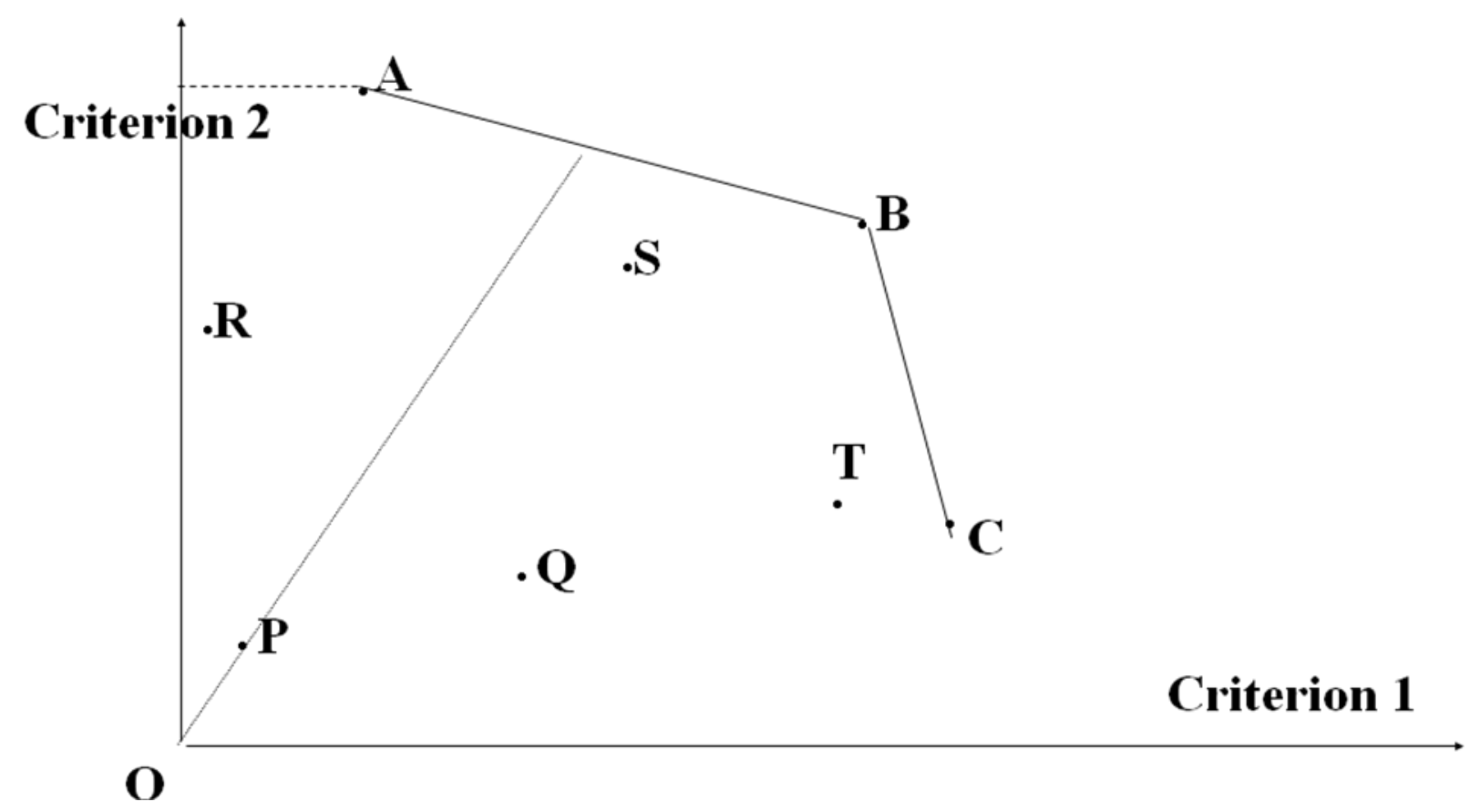

\section{FIGURE 1}

\section{Having a common set of weights (with an upper limit to the overall score) means that a line such as $\mathrm{AB}$ or $\mathrm{BC}$ acts as the frontier. The slope of such a line determines the weights attached to the criteria.}

Now consider what happens when alternative $\mathrm{P}$ is removed from further consideration (possibly on the grounds that it is clearly not a suitable contender in some selection problem). Q now has the lowest score. This makes facet BC (extended) to act as the new linear frontier. Unit A was previously ranked first equal (maximum score), but now it slides down the rankings below all of B, C, T, S and Q! Karsak and Ahiska (2005) used the maximin method in a selection problem: to choose a particular piece of equipment from a number of competing alternatives. Expressed in these terms the removal of a point such as $\mathrm{P}$ corresponds to removing an irrelevant alternative - one that would never be selected because of its poor performance. Yet its removal causes huge changes in the rankings. This violates the axiom of decision theory known as Sen's property alpha (Sen, 1969), also known as the Chernoff condition (Chernoff, 1954), which states that the removal or addition of an irrelevant alternative should not affect the decision. The selection decision should be independent of irrelevant alternatives. The removal of such unwanted points could for example arise in an initial screening stage, where alternatives which do not measure up to certain minimum standards are removed from further consideration. They could also be removed from simple dominance arguments. A memorable illustration of the principle is an anecdote attributed to the philosopher Sidney Morgenbesser:

After finishing dinner, Sidney Morgenbesser decides to order dessert. The waitress tells him he has two choices: apple pie and blueberry pie. Sidney orders the apple pie. After a few minutes 
the waitress returns and says that they also have cherry pie, at which point Morgenbesser says "In that case I'll have the blueberry pie."

A variation on the above which deals with multiple inputs as well as multiple outputs is due to Chiang and Tzeng (2000). They employ a fuzzy programming approach where the membership function "refers to the level of achievement of the efficiency ratio for the decision making unit". This replaces the piecewise linear frontier of DEA by a single linear frontier with fewer units located upon it and thus fewer $100 \%$ efficiency ratings.

Stewart (1996) considers a form of DEA which is based on the difference between a weighted sum of inputs and a weighted sum of outputs, rather than a ratio of these aggregates. He demonstrates an important equivalence between these two forms, and points out that this provides useful linkage with multi-criteria decision analysis where such value functions are commonly used as measures of worth. Then in an effort to get away from the extreme sets of weights which can arise in DEA, the minimax deviation from the frontier is considered as the objective. Compared to other ways of obtaining common weights, Stewart argues that "the min-max form of objective tends to find value functions on which a number of efficient DMUs will have the same value, and thus tends to characterize the efficient frontier better". However, he wisely observes that whatever bounds are placed on the weights "will take on an importance out of proportion to the rather 'fuzzy' nature of their assessment". Consequently he recommends the application of sensitivity analysis to the weights.

Another attempt at linking multi-criteria decision analysis and DEA is due to Li and Reeves (1999). They take that view that efficiency evaluation should be extended beyond a single measure. But unlike the profiling approach of Tofallis (1997) in which different aspects of performance are evaluated separately, they choose to include all variables in a single data envelopment analysis which is then complemented by two additional measures: one of these is the maximin approach and the other takes as its objective the maximization of the sum of the efficiency scores. This is then viewed as a multi-objective programming problem with three objective functions. An element of sensitivity analysis is thus afforded. Climaco et al (2008) apply the TRIMAP interactive software to this problem; they interpret the two additional objectives as representing equity and general benevolence.

Troutt, Zhang and Pettypool (1993) use the maximin rule as a way of further ranking those alternatives which have all been given the same $100 \%$ efficiency score from a data envelopment analysis. This differs from the above approaches in that only efficient alternatives are considered at this second stage. Hence the worst performers cannot influence the resulting weights and so is a definite improvement. The alternatives which will now influence the position of the linear frontier will be those that are at the ends of the frontier. In a two dimensional setting these will be points $\mathrm{A}$ and $\mathrm{C}$ in Figure 1, but in higher dimensions they will be the points on the perimeter of the observed frontier. Such points have very high scores in one criterion but are weak in the others, and are sometimes referred to as 'mavericks'. They contrast with good all-rounders. One might 
also include in this second stage those alternatives which are Pareto-optimal even though they do not appear on the convex hull, for example point D in Figure 2. Such points are also 'good all-rounders' but find themselves behind the frontier.

Troutt (1997, and references therein) has written a number of papers applying the maximin approach to DEA with both multiple inputs and multiple outputs. He calls the resulting scores the MER - the maximin efficiency ratios. He makes the following observation:

When the MER model was first discussed (without subsequent benefit of theoretical justification), some critics argued that "optimal" multipliers should not be based on least efficient units. While that criticism has intuitive merit, it may be noted that a reverse perspective is actually more fruitful. Namely, the minimum efficiency, as well as the average (or any other summary statistic) depends on the weights. Such weights or multipliers may, or may not, in general, maximize the likelihood of the resulting aggregate measure. Thus, from the maximum likelihood perspective the procedure appears intuitive. However, this apparent "contradiction of intuitions" continues to be interesting and not yet fully resolved.

Troutt and Zhang (1993) also note that "a possible objection is that the resulting weights may be overly influenced by the worst performers". They try to address this by saying "choices of weights which increase the minimum ratio frequently increase the average ratio as well, and conversely. Hence the maximin aggregation principle appears similar in expected performance to maximization of the average, which clearly depends on the performance data of the whole set of [DMUs]". This is not a persuasive argument because in the maximization of the average each point has equal influence, whereas in the maximin case this is far from being true. They also try to address the issue by first noting that using maximin leads to all scores being squeezed into the narrowest range - which is true. It is then argued that the range is a measure of dispersion, as is the variance, so one would expect similar results to minimizing the variance of the scores. Once again, the conclusion does not follow because the calculation of variance is based on all observations whereas the range is not. Moreover, the range statistic is not merely sensitive to outliers, it is entirely determined by them.

To help us understand why we would not expect similar scoring performance let us draw some parallels with methods of fitting models to data. Consider the deviations from the $100 \%$ score as being residuals, and consider that we are fitting a linear model which is constrained not to have any data points lying above it. It now becomes clear that the maximin approach corresponds to fitting using the Chebyshev or $\mathrm{L}_{\infty}$ norm, and the minimization of the average residual corresponds to the $\mathrm{L}_{1}$ norm. It is well established that these fitting approaches produce very different models and so we cannot expect to obtain similar performance as claimed above. Specifically, the $\mathrm{L}_{1}$ norm is less sensitive to outliers than least squares regression, whereas the Chebyshev norm is more sensitive to outliers than least squares. 


\section{Can the maximin approach produce a single winning alternative?}

Pettypool and Karathanos (2004) propose the maximin approach for reward systems where there are multiple measures of reward and contribution involved. They provide a numerical example which includes three reward measures (outputs) and two contribution measures (inputs). Despite the fact that there are only seven alternatives, maximin still does not produce a single winner. Looking at Figure 1 would seem to indicate that in the case of two outputs there will normally be three alternatives which will appear at the extremes of the score range. This is because the frontier line needs to come in as close as possible to the data points in order to keep the score range narrow. In this case $\mathrm{P}$ gets the lowest score, with A and B getting the highest score. As the number of criteria is increased, the higher dimensionality of the problem means that the frontier will have more dimensions and so more observations will lie upon it. Hence, although having a single set of common criteria weights will reduce the number scoring $100 \%$, we cannot rely on the maximin approach to produce a single winner.

\section{Criteria can be completely ignored}

Consider the set of alternatives displayed in Figure 2. In this case $\mathrm{R}$ will have the lowest score as it has the worst performance on both criteria. Its score will be maximised by referring to the horizontal dashed line as a frontier. $\mathrm{R}$ is not fully enveloped by a pair of frontier units in the way that $\mathrm{P}$ was in Figure 1, and this causes difficulties. We shall now show that using the extension of this horizontal line as a frontier to assess all other alternatives leads to criterion 1 being completely ignored in the assessment i.e. a zero weight will be applied. The demonstration involves the similar right-angled triangles $\mathrm{R}^{\prime} \mathrm{Y}_{\mathrm{A}} \mathrm{O}$, and $\mathrm{RY} \mathrm{Y}_{\mathrm{R}} \mathrm{O}$. The angle subtended at the origin is the same for both triangles, and the cosine of this angle equates to $O Y_{R} / O R=O Y_{A} / O R '$. Therefore $O R / O R '=O Y_{R} / O Y_{A}$. But OR/OR' is precisely the score for $\mathrm{R}$ and $\mathrm{OY}_{\mathrm{R}} / \mathrm{OY}_{\mathrm{A}}$ is the ratio of values on criterion 2 . Thus the values on criterion 1 play no part in the assessment of $R$. The same argument applies to the assessment of the other alternatives. 


\section{Criterion 2}

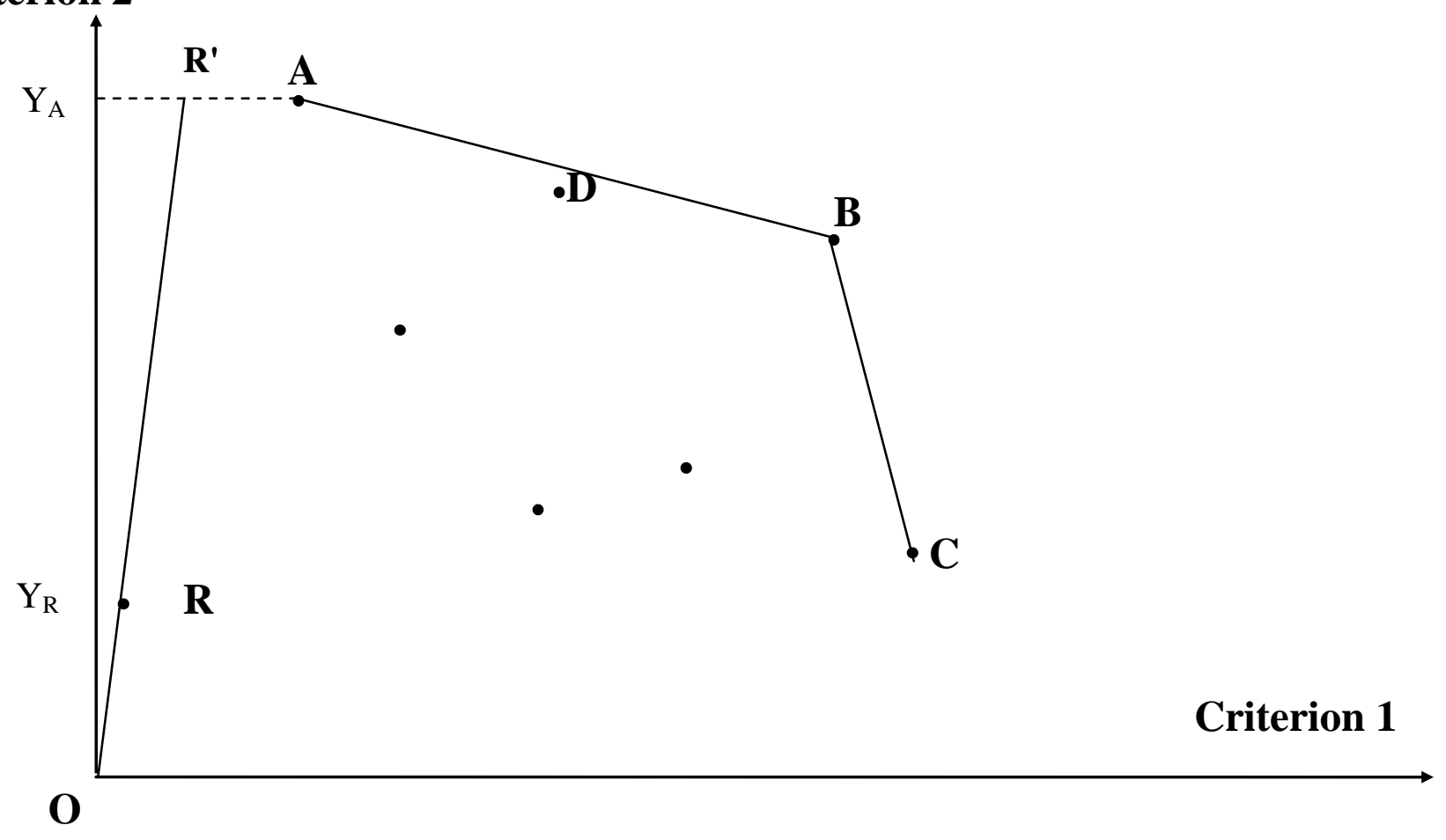

Figure 2

When we attempt to project alternative $R$ onto the frontier we find that its 'target' $\left(R^{\prime}\right)$ does not lie between observed efficient units - i.e. it is not naturally enveloped. This leads to a horizontal frontier and a zero weight for criterion 1.

\section{Conclusion}

At first sight using the maximin rule to choose a set of common weights might seem an attractive approach to an analyst. One reason is that it is not subjective, but more importantly, it reduces the likelihood of being confronted by those who fare badly from the resulting rankings - this is because the method focuses on raising their score. Thus the analyst may be able to avoid having to argue with low scorers about the weights chosen.

However, this paper has shown that a number of serious drawbacks arise when using this rule in assessing performance. Any choice of weights corresponds to deciding how much each criterion is worth in terms of utility or value. It is clear that the maximin rule is allowing these utility values to be determined by the worst performers. This is as sensible 
as allowing the worst performing student to decide how much weight to attach to each of the various assessments taken by the class.

Next consider the problem of selecting from a set of alternatives. To ease the decision a common way to reduce the number of alternatives is to use screening or filtering. This is simply the removal of those alternatives which are clearly inadequate because they do not meet certain minimal standards. This step is carried out to enable the decision maker to focus attention on the serious contenders and should not affect the final decision.

However, when used in conjunction with the maximin rule such a process will remove the worst performers and so lead to a different set of weights and a different ranking of the remaining alternatives. Decisions based on the maximin rule are highly sensitive to the inclusion or exclusion of alternatives whose performance is so poor as to be completely irrelevant to the selection decision.

We also showed that when the worst performing alternative is not naturally enveloped by units on the frontier (a common occurrence with real data), then certain criteria will be given zero weight and so be completely ignored in the analysis. Given that the criteria will have been carefully selected as being appropriate at the start, it is strange that they are now effectively being dismissed.

Whilst, the maximin approach has been used in the allocation of resources in order to reduce inequality, its use to assess such a situation of need is a different matter entirely. The stage of evaluation to determine who is most in need or most deserving is separate from the stage of assigning resources or rewards. Rawls' difference principle may or may not be of use in the allocation stage but it is definitely not appropriate in the assessment stage. To persist in using it for both would be to minimise the perceived need of the worst off and thereby reduce the resources allocated to them. 


\section{REFERENCES}

Butler, M and Williams, HP (2002). Fairness versus efficiency in charging for the use of common facilities. J Opl Res Soc, 53, 1324-1329.

Chernoff, H (1954). Rational selection of decision functions. Econometrica, 22, 423-443.

Chiang, CI and Tzeng, GH (2000). A multiple objective programming approach to data envelopment analysis. In New Frontiers of Decision making for the Information Technology Era, eds Yong Shi and Milan Zeleny. World Scientific, Singapore.

Climaco, JCN, de Mello, JCS and Meza LA (2008). Performance measurement: From DEA to MOLP. In Encyclopedia of Decision Making and Decision Support Technologies, eds. Frédéric Adam and Patrick Humphreys.

Du, Ding-Zhu (1996). Minimax and its applications. In Handbook of Global Optimization, p.339-367. Horst, R and Pardalos, PM (eds). Kluwer Academic, Dordrecht.

Farahani, RZ, Seifi, MS, and Asgari, N (2010). Multiple criteria facility location problems: A survey. Applied Mathematical Modelling, forthcoming.

Karsak, EE (2004). A Practical Common Weight MCDM Approach for Emerging Market Selection", Proceedings of the 34th International Conference on Computers and Industrial Engineering (ICC\&IE), San Francisco, California, USA, 159-164.

Karsak, EE and Ahiska, SS (2005). Practical common weight multi-criteria decisionmaking approach with an improved discriminating power for technology selection. Int. J. Production Research. 43 (8), 1537-1554.

Li, X-B and Reeves, GR (1999). A multiple criteria approach to data envelopment analysis. Eur J Oper Res 115, 507-517.

Ogryczak, W (1997). On the lexicographic minimax approach to location problems. Eur J Oper Res 100, 566-585.

Ogryczak, W and Sliwinski, T (2002). On equitable approaches to resource allocation problems: the conditional minimax solutions. J Telecommunications and IT 3/2002,4-48.

Pettypool, MD and Karathanos, P (2004). An equity check. Eur J Oper Res 157, 465-470.

Rardin, RL (1998). Optimization in Operations Research. Prentice Hall, New Jersey.

Rawls, JA (1999). A Theory of Justice. Revised edition. Cambridge, MA: Harvard University Press.

Sen, AK (1969). Quasi-transitivity, rational choice and collective decisions. Review of Economic Studies, 36, 381-393. 
Stewart, TJ (1996). Relationships between data envelopment analysis and multicriteria decision analysis. J Oper Res Soc 47, 654-665.

Tofallis, C (1997). Input Efficiency Profiling: An Application to Airlines. Computers and Operations Research 24 (3), 253-258.

Troutt, MD, (1997). Derivation of the maximin efficiency ratio model from the maximum decisional efficiency principle. Annals of Ops Res, 73, 323-338.

Troutt, MD and Zhang, A, (1993). The maximin efficiency ratio as a one step heuristic ranking device - comparison to two modified DEA analyses. Working paper, Dept of Management, Southern Illinois University, Carbondale, Illinois.

Troutt, MD, Zhang, A, and Pettypool, MD (1993). A maximin efficiency ratio model for consensus weights and extended ranking of technically efficient units. Working paper, Dept of Management, Southern Illinois University, Carbondale, Illinois.

Yaari, ME and Bar-Hillel, M (1984). On dividing justly. Social Choice and Welfare, 1, 124 